\title{
Publisher's Note: Detecting monopole charge in Weyl semimetals via quantum interference transport [Phys. Rev. B 93, 161110(R) (2016)]
}

Xin Dai, Hai-Zhou Lu, Shun-Qing Shen, and Hong Yao (Received 29 April 2016; published 9 May 2016)

DOI: 10.1103/PhysRevB.93.199902

This paper was published online on 18 April 2016 with an error in Ref. [27]. Reference [27] should read as "C.-L. Zhang, S.-Y. Xu, I. Belopolski, Z. Yuan, Z. Lin, B. Tong, G. Bian, N. Alidoust, C.-C. Lee, S.-M. Huang, T.-R. Chang, G. Chang, C.-H. Hsu, H.-T. Jeng, M. Neupane, D. S. Sanchez, H. Zheng, J. Wang, H. Lin, C. Zhang, H.-Z. Lu, S.-Q. Shen, T. Neupert, M. Z. Hasan, and S. Jia, Nat. Commun. 7, 10735 (2016)." The reference has been corrected as of 29 April 2016. The reference is correct in the printed version of the journal. 